\title{
Brentuximab Vedotin
}

\section{Abstract}

\section{Introduction}

Brentuximab vedotin is an anticancer antibodydrug conjugate (ADC) product under development by Seattle Genetics Inc. (Bothell, WA, USA) and its licensee Millennium: The Takeda Oncology Company. The ADC comprises the antiCD30 monoclonal antibody cAC10 conjugated to the cytotoxic agent monomethyl auristatin $\mathrm{E}$ (MMAE), a synthetic analog of the tubulin polymerization inhibitor dolastatin 10 . The chimeric antibody is covalently coupled to MMAE through a valine-citrulline peptide linker. Brentuximab vedotin is designed to be stable in the bloodstream, but to release MMAE upon internalization into CD30-expressing tumor cells, resulting in a targeted cell-killing effect. The CD30 antigen is highly expressed by a variety of hematologic malignancies, including Hodgkin lymphoma and some T-cell non-Hodgkin lymphomas. Clinical development for the treatment of Hodgkin lymphoma and non-Hodgkin lymphoma (specifically anaplastic large cell lymphoma [ALCL]) is being conducted in North America and Europe.

\subsection{Company Agreements}

In December 2009, Seattle Genetics Inc. and Millennium: The Takeda Oncology Company, a wholly owned subsidiary of Takeda Pharmaceutical Company Limited, entered into a collaboration agreement to globally develop and commercialize brentuximab vedotin. Under the collaboration, Seattle Genetics Inc. will receive an upfront payment of \$US60 million and retains full commercialization rights for brentuximab vedotin in the US and Canada. The Takeda Group will have exclusive rights to commercialize the product candidate in all countries other than the US and Canada. Seattle Genetics Inc. is entitled to receive progress and sales-dependent milestone payments in addition to tiered doubledigit royalties based on net sales of brentuximab vedotin within the Takeda Group's licensed

This drug profile has been extracted from Wolters Kluwer's $A d i s^{\mathrm{TM}} R \& D$ Insight drug pipeline database. $R \& D$ Insight tracks and evaluates drug development worldwide through the entire development process, from discovery, through pre-clinical and clinical studies to market launch. This is an open access article published under the terms of the Creative Commons License "Attribution-NonCommercial-NoDerivative 3.0" (http://creative commons.org/licenses/by-nc-nd/3.0/) which permits non-commercial use, distribution, and reproduction, provided the original work is properly cited and not altered. 
territories. Milestone payments to Seattle Genetics Inc. could total more than \$US230 million. Seattle Genetics Inc. and the Takeda Group will jointly fund worldwide development costs on a 50:50 basis. Development funding by the Takeda Group over the first 3 years of the collaboration is expected to be at least \$US75 million. In Japan, the Takeda Group will be solely responsible for development costs. ${ }^{[1]}$

An agreement between Seattle Genetics Inc. and Albany Molecular Research for the current good manufacturing practice (cGMP) of its proprietary drug-linker system was established in May 2005. The arrangement also secures rights for ADC licensees of Seattle Genetics Inc. to work directly with Albany Molecular Research to obtain cGMP clinical trial supplies of drug-linker units. ${ }^{[2,3]}$

\subsection{Key Development Milestones}

Seattle Genetics Inc. plans to submit a biologics license application (BLA) to the US FDA in the first quarter of 2011. The BLA will aim to seek approval for both relapsed or refractory Hodgkin lymphoma and relapsed or refractory systemic ALCL. In Europe, Millennium: The Takeda Oncology Company has initiated discussions with regulators to support the submission of a marketing authorization application (MAA) to the European Medicines Agency (EMA) in the first half of 2011. ${ }^{[4]}$

The US FDA and the EMA have granted orphan drug designation to brentuximab vedotin for the treatment of Hodgkin lymphoma and ALCL (a type of non-Hodgkin lymphoma). ${ }^{5,6]}$ In March 2009, the FDA granted fast-track designation to brentuximab vedotin for the treatment of Hodgkin lymphoma. ${ }^{[7]}$

\subsubsection{Hodgkin Lymphoma}

Seattle Genetics Inc. and Millennium: The Takeda Oncology Company have initiated a phase III trial (AETHERA; NCT01100502) of brentuximab vedotin in patients at high risk of residual Hodgkin lymphoma following autologous stem cell transplant (ASCT). This randomized, double-blind, placebo-controlled trial is evaluating the safety and efficacy of brentuximab vedotin plus best supportive care (BSC) compared with placebo plus BSC. Approximately 322 patients are being enrolled at sites in the US and Europe. The primary outcome measure of the trial is progression-free survival (PFS), and secondary outcomes measures include overall survival, safety, and tolerability. Patients will receive brentuximab vedotin every 3 weeks for up to approximately 1 year. For the purposes of the trial, high-risk patients are defined as those with a history of refractory Hodgkin lymphoma, those who relapse or progress within 1 year of receiving first-line chemotherapy, and/or those who have disease outside of the lymph nodes at the time of pre-ASCT relapse. ${ }^{[8]}$ Interim results were presented in December 2010. Seattle Genetics Inc. plans to submit a BLA in the first quarter of 2011 for approval of brentuximab vedotin in relapsed or refractory Hodgkin lymphoma and systemic ALCL. Millennium: The Takeda Oncology Company plans to submit an MAA in the first half of 2011. Additionally, a limited patient access program for qualified patients will be set up in the US in early 2011. Outside of the US and Canada, brentuximab vedotin will be available to qualified patients through a Named Patient Programme. ${ }^{[9]}$

Seattle Genetics Inc. and Millennium: The Takeda Oncology Company initiated a phase II/ III study (NCT01196208) of brentuximab vedotin in patients with Hodgkin lymphoma who received placebo in the phase III AETHERA trial (SGN35-0005). The trial expects to enroll up to 80 patients by invitation only and aims to be completed by December 2011.

In February 2009, Seattle Genetics Inc. initiated a single-arm pivotal phase II trial (NCT 00848926) to assess the efficacy and safety of single-agent brentuximab vedotin $(1.8 \mathrm{mg} / \mathrm{kg}$ every 3 weeks) in 102 patients with relapsed or refractory Hodgkin lymphoma under a special protocol assessment agreement. The primary endpoint of the study will be objective response rate and secondary endpoints to include duration of response, PFS, overall survival, and tolerability. The company completed enrollment of patients at more than 30 sites in the US, Canada, and Europe in August 2009. Top-line data were 
reported by Seattle Genetics Inc. and Millennium: The Takeda Oncology Company in September 2010. ${ }^{[10]}$

In July 2009, Seattle Genetics Inc. initiated a multicenter, phase II trial (NCT00947856) investigating the efficacy and tolerability of retreatment with brentuximab vedotin $(1.8 \mathrm{mg} / \mathrm{kg}$ every 3 weeks) in patients with relapsed or refractory Hodgkin lymphoma or systemic ALCL who had previously responded to brentuximab vedotin. The trial is expected to enroll 125 patients and is taking place in the US and Europe. ${ }^{[1,12]}$

In January 2010, Seattle Genetics Inc. and Millennium: The Takeda Oncology Company initiated an open-label, single-arm phase I trial (SGN35-009; NCT01060904) of brentuximab vedotin in combination with ABVD (doxorubicin, bleomycin, vinblastine and dacarbazine) in the treatment of patients with newly diagnosed Hodgkin's lymphoma. The dose-escalation trial will enroll 40 patients in the US and Canada. ${ }^{[13]}$

\subsubsection{Non-Hodgkin Lymphoma (including Anaplastic Large Cell Lymphoma)}

In June 2009, Seattle Genetics Inc. initiated a pivotal phase II study (NCT00866047) of singleagent brentuximab vedotin $(1.8 \mathrm{mg} / \mathrm{kg}$ every 3 weeks) in patients with relapsed or refractory systemic ALCL. Recruitment of 58 patients from centers in the US, Canada, and the EU was completed in May 2010. Preliminary, top-line results have been reported. ${ }^{[14-17]}$

In July 2009, Seattle Genetics Inc. initiated a multicenter, phase II trial (NCT00947856) investigating the efficacy and tolerability of retreatment with brentuximab vedotin $(1.8 \mathrm{mg} / \mathrm{kg}$ every 3 weeks) in patients with systemic ALCL or relapsed or refractory Hodgkin lymphoma who had previously responded to brentuximab vedotin. The trial is expected to enroll 125 patients and is taking place in the US and Europe. ${ }^{[11,12]}$

\subsubsection{General Hematologic Malignancies}

Seattle Genetics Inc. has conducted a phase I trial (NCT00430846) of brentuximab vedotin (given every 21 days) in 44 refractory patients with Hodgkin lymphoma or CD30-positive hematologic malignancies. The trial evaluated the safety and pharmacokinetics of brentuximab vedotin among patients enrolled at multiple centers throughout the US. Data presented in December 2008 demonstrated multiple complete and partial responses at well tolerated doses. ${ }^{[18-20]}$

Seattle Genetics Inc. and Millennium: The Takeda Oncology Company are also conducting a phase I study to assess the cardiac safety of brentuximab vedotin in patients with CD30positive hematologic cancers (NCT01026233). ${ }^{[21]}$ The study was expected to be completed in August 2010 but as of February 2011, the companies have completed enrollment of 40 patients and the trial is ongoing.

A phase I trial (NCT00649584) was initiated in the US in March 2008, to evaluate brentuximab vedotin in 72 patients with refractory or relapsed CD30-positive hematologic malignancies (Hodgkin lymphoma or systemic ALCL). The study was expected to be completed in December 2010. However, this trial was terminated as Seattle Genetics Inc. decided not to enroll cohorts of combined brentuximab vedotin and gemcitabine therapy. ${ }^{[17,22-24]}$

\subsection{Patent Information}

The US Patent and Trademark Office has issued Seattle Genetics Inc. with a patent related to its ADC technology. US Patent No. 7659241 , covers cleavable linkers and potent auristatin drug payloads used in certain Seattle Genetics Inc. ADC programs, including brentuximab vedotin. ${ }^{[25]}$

Seattle Genetics Inc. was issued a US patent covering the cell-killing component of brentuximab vedotin in May 2005. ${ }^{[3]}$

\section{Scientific Summary}

\subsection{Pharmacokinetics}

Phase I: Area under the concentration-time curve (AUC) increased relative to dose level of brentuximab vedotin in this phase I trial. The trial enrolled patients with relapsed or refractory CD30positive lymphomas $(n=37)$ and brentuximab vedotin was administered weekly for 3 weeks in 28 -day treatment cycles at doses of $0.4-1.4 \mathrm{mg} / \mathrm{kg}$ (30 minute or 2 hour intravenous infusions). [26] 
Table I. Features and properties

\begin{tabular}{ll}
\hline $\begin{array}{l}\text { Alternate names } \\
\text { Originator }\end{array}$ & CAC10-vcMMAE; SGN 35; SGN-35 \\
Licensee(s) & Seattle Genetics Inc. \\
Highest development phase & III (Europe, US) \\
Active development indications & Hodgkin lymphoma, Non-Hodgkin lymphoma \\
Class & Auristatins, drug conjugates, monoclonal antibodies \\
Mechanism of action & Tubulin polymerization inhibitors \\
CAS Registry number & 914088-09-8 \\
Route of administration & IV \\
Pharmacodynamics & Potent cytotoxic activity against CD30-expressing cells in vitro; associated with disease-free \\
& survival mouse Hodgkin lymphoma model; dose-dependent antitumor activity in preclinical \\
models of anaplastic large cell lymphoma; has superior antitumor efficacy compared with non- \\
targeted drugs, in preclinical models of Hodgkin lymphoma \\
ATC codes & L01 (Antineoplastic Agents), L01X-C (Monoclonal antibodies) \\
WHO ATC code & L1 (Antineoplastics), L1X3 (Antineoplastic monoclonal antibodies) \\
EphMRA ATC code & Alopecia, diarrhea, fatigue, fever, injection-site reactions, musculoskeletal pain, nausea, \\
Adverse events & neutropenia, peripheral nervous system diseases, thrombocytopenia \\
Occasional &
\end{tabular}

AUC increased relative to dosage and did not accumulate with repeated dosing in a phase I dose-escalation trial in patients with hematologic malignancies. ${ }^{\text {[27] }}$

Preclinical: The elimination half-life of brentuximab vedotin in mice was approximately 5 days and the maximum tolerated dose was $>30 \mathrm{mg} / \mathrm{kg}$. ${ }^{[28]}$

\subsection{Adverse Events}

Phase III: In the interim results from the ATHERA trial of brentuximab vedotin in patients at high risk of residual Hodgkin lymphoma following ASCT, the most common adverse events were peripheral sensory neuropathy $(47 \%)$, fatigue (46\%), nausea (42\%), upper respiratory tract infection $(37 \%)$, and diarrhea (36\%). The most common grade 3 or 4 adverse events were neutropenia (20\%), peripheral sensory neuropathy (8\%), thrombocytopenia (8\%), and anemia $(6 \%) .{ }^{[9]}$

Phase II: Brentuximab vedotin demonstrated a similar safety and tolerability profile to prior clinical studies in a phase II trial of 102 patients with relapsed or refractory Hodgkin lymphoma. In this open-label trial, patients received bren- tuximab vedotin $(1.8 \mathrm{mg} / \mathrm{kg})$ every 3 weeks for a maximum of 16 doses. ${ }^{[10]}$

Brentuximab vedotin was associated with manageable adverse events in a phase II clinical trial in 58 patients with relapsed or refractory systemic ALCL. The most common adverse events were nausea (38\%), peripheral neuropathy (38\%), fatigue (34\%), fever (33\%), and diarrhea (29\%). The most common grade 3 or higher adverse events were neutropenia (21\%), peripheral neuropathy $(10 \%)$, thrombocytopenia (14\%), and anemia $(7 \%) \cdot{ }^{[4]}$

Phase I: In a phase I trial, brentuximab vedotin exceeded a maximum tolerated dose at $1.4 \mathrm{mg} / \mathrm{kg}$ and exhibited grade 3 dose-limiting toxicities of diarrhea and vomiting, and hyperglycemia grade 4 . The most common drug-associated adverse events were peripheral neuropathy, nausea, fatigue, diarrhea, dizziness, and neutropenia; most were grade 1 or 2 in severity. The trial enrolled patients with relapsed or refractory CD30-positive lymphomas $(n=37)$ and brentuximab vedotin was administered weekly for 3 weeks in 28-day treatment cycles at doses of $0.4-1.4 \mathrm{mg} / \mathrm{kg}$ (30 minute or 2 hour intravenous infusions). ${ }^{[26]}$

Brentuximab vedotin was generally well tolerated in a phase I trial among 44 evaluable 
Table II. History

\begin{tabular}{llll}
\hline Event Date & Update type & Comment & Update date \\
\hline 7 December 2010 & Scientific Update & Efficacy and adverse events data from a phase II trial in & 11 December 2010 \\
& & anaplastic large cell lymphoma presented at the 52nd Annual & \\
& Meeting and Exposition of the American Society of Hematology \\
& $(\text { ASH-2010 })^{[4]}$
\end{tabular}

6 December $2010 \quad$ Scientific Update

5 December 2010

Scientific Update

11 October 2010

Scientific Update

27 September 2010

Scientific Update

8 June 2010

Scientific Update

24 May 2010

30 April 2010

10 April 2010

30 March 2010

30 March 2010

29 January 2010

Phase Change

29 January 2010

Phase Change

16 December 2009

Licensing Status

8 December 2009

Scientific Update

5 December 2009
Scientific Update
Interim efficacy and adverse events data from the phase III AETHERA trial in Hodgkin lymphoma presented at the 52nd Annual Meeting and Exposition of the American Society of Hematology (ASH-2010) ${ }^{[9]}$

Additional efficacy data from a phase II trial in Hodgkin lymphoma presented at the 52nd Annual Meeting and Exposition of the American Society of Hematology (ASH-2010) ${ }^{[37]}$

Efficacy data from a phase II trial (NCT00866047) in relapsed and refractory non-Hodgkin lymphoma (anaplastic large cell lymphoma) released by Seattle Genetics Inc. and Millennium: The Takeda Oncology Company ${ }^{[14]}$

Efficacy data from a pivotal phase II trial in relapsed and refractory Hodgkin lymphoma released by Seattle Genetics Inc. and Millennium: The Takeda Oncology Company[10]

Interim efficacy and adverse events data from a clinical trial in Hodgkin lymphoma and systemic anaplastic large cell lymphoma (non-Hodgkin lymphoma) presented at the 46th Annual Meeting of the American Society of Clinical Oncology (ASCO-2010) $^{[31]}$

Seattle Genetics Inc. completes enrollment in its phase II trial for relapsed/refractory anaplastic large cell lymphoma in the US, EU, and Canada

Seattle Genetics Inc. terminates the phase I trial of brentuximab alone and in combination with gemcitabine for CD30-positive hematologic malignancies

InThought Forecasts

inThought Analysis for Hodgkin lymphoma updated

Phase III clinical trials in Hodgkin lymphoma (patients at high risk of residual Hodgkin lymphoma following autologous stem cell transplant) in Europe (IV)

Phase III clinical trials in Hodgkin lymphoma (patients at high risk of residual Hodgkin lymphoma following autologous stem cell transplant) in the US (IV)

Phase I clinical trials (combination therapy) in Hodgkin lymphoma in Canada (IV)

Phase I clinical trials (combination therapy) in Hodgkin lymphoma in the US (IV)

Seattle Genetics Inc. enters into a licensing agreement with Millennium: The Takeda Oncology Company ${ }^{[1]}$

Efficacy, pharmacokinetic, and adverse events data from a phase I trial in Hodgkin lymphoma and non-Hodgkin lymphoma presented at the 51st Annual Meeting and Exposition of the American Society of Hematology (ASH-2009) ${ }^{[26]}$

Updated interim efficacy and adverse events data from a phase I trial in Hodgkin lymphoma and CD30-positive lymphoma presented at the 51st Annual Meeting and Exposition of the American Society of Hematology (ASH-2009)
9 December 2010

7 December 2010

12 October 2010

1 October 2010

15 June 2010

26 May 2010

19 May 2010

10 April 2010

15 February 2011

30 March 2010

5 February 2010

5 February 2010

17 December 2009

9 December 2009

31 December 2009

Continued next page 
Table II. Contd

\begin{tabular}{llll}
\hline Event Date & Update type & Comment & Update date \\
\hline 8 September 2009 & Scientific Update & $\begin{array}{l}\text { Further updated efficacy data from a phase I trial in Hodgkin } \\
\text { lymphoma and CD30-positive hematologic malignancies } \\
\text { released by Seattle Genetics Inc. }{ }^{[29]}\end{array}$ & 16 September 2009 \\
& & S &
\end{tabular}

25 August $2009 \quad$ Trial Update

25 July 2009

Trial Update

23 July 2009

Scientific Update

19 June 2009

Phase Change

2 June 2009

Scientific Update

31 March 2009

Regulatory Status

19 March 2009

19 March 2009

20 February 2009

20 February 2009

20 February 2009

20 February 2009

27 January 2009

27 January 2009

27 January 2009

8 December 2008

25 October 2008

Scientific Update

24 October 2008

Scientific Update

4 June 2008

Scientific Update

3 June 2008

Scientific Update

Phase Change

Phase Change

Phase Change

Phase Change

Phase Change

Trial Update

Regulatory Status

Regulatory Status

Regulatory Status

Scientific Update
Seattle Genetics Inc. completes enrollment in its phase II trial for brentuximab vedotin in Hodgkin lymphoma in the US, Canada, and Europe

Seattle Genetics Inc. initiates a phase II trial of retreatment in relapsed/refractory Hodgkin lymphoma and systemic anaplastic large cell lymphoma in the US ${ }^{[1]}$

Pharmacodynamics data from a preclinical trial in Hodgkin lymphoma presented at the 100th Annual Meeting of the American Association for Cancer Research (AACR-2009) ${ }^{[36]}$

Phase II clinical trials in non-Hodgkin lymphoma in Canada (IV)

Interim efficacy and adverse events data from a phase I trial in hematologic malignancies presented at the 45th Annual Meeting of the American Society of Clinical Oncology (ASCO-2009) $^{[23]}$

Brentuximab vedotin receives fast-track designation for Hodgkin lymphoma (IV, infusion) in the US

Phase II clinical trials in non-Hodgkin lymphoma in the EU (IV)

Phase II clinical trials in non-Hodgkin lymphoma in the US (IV)

Phase II clinical trials in Hodgkin lymphoma in Canada (IV)

Phase II clinical trials in Hodgkin lymphoma in Europe (IV)

Phase II clinical trials in Hodgkin lymphoma in the US (IV)

Seattle Genetics Inc. initiates enrollment in a phase II trial for Hodgkin lymphoma in Canada, Europe, and the US

Brentuximab vedotin receives orphan drug status for non-Hodgkin lymphoma in the EU

Brentuximab vedotin receives orphan drug status for Hodgkin lymphoma in the EU

Brentuximab vedotin receives orphan drug status for non-Hodgkin's lymphoma in the US

Updated efficacy data from a phase I trial in hematologic malignancies presented at the 50th Annual Meeting and Exposition of the American Society of Hematology $(\mathrm{ASH}-2008)^{[18]}$

Interim efficacy data from a phase I trial in hematologic malignancies presented at the 20th-EORTC-NCI-AACR Symposium on Molecular Targets and Cancer Therapeutics (EORTC-NCI-AACR-2008) ${ }^{[19]}$

Pharmacodynamic data from a preclinical trial in hematologic malignancies presented at the 20th-EORTC-NCI-AACR Symposium on Molecular Targets and Cancer Therapeutics (EORTC-NCl-AACR-2008) (35] $^{[3}$

Interim efficacy and adverse events data from a phase I trial in Hodgkin lymphoma presented at the 44th Annual Meeting of the American Society of Clinical Oncology (ASCO-2008) ${ }^{[30]}$

Pharmacokinetics data from a phase I trial in Hodgkin lymphoma presented at the 44th Annual Meeting of the American Society of Clinical Oncology (ASCO-2008) ${ }^{[27]}$
31 August 2009

28 July 2009

23 July 2009

23 June 2009

8 June 2009

1 April 2009

19 June 2009

19 June 2009

23 February 2009

23 February 2009

23 February 2009

23 February 2009

28 January 2009

28 January 2009

28 January 2009

10 December 2008

28 October 2008

12 November 2008

11 June 2008

18 June 2008

Continued next page 
Table II. Contd

\begin{tabular}{|c|c|c|c|}
\hline Event Date & Update type & Comment & Update date \\
\hline 31 March 2008 & Trial Update & $\begin{array}{l}\text { Seattle Genetics Inc. initiates enrollment in a second phase I } \\
\text { trial for refractory or relapsed CD30-positive hematologic } \\
\text { malignancies in the US }\end{array}$ & 2 April 2008 \\
\hline 8 November 2007 & Scientific Update & $\begin{array}{l}\text { Interim results from a phase I clinical trial in patients with } \\
\text { hematologic malignancies added to the adverse events and } \\
\text { cancer therapeutic trials sections }\end{array}$ & 8 November 2007 \\
\hline 15 February 2007 & Regulatory Status & $\begin{array}{l}\text { Brentuximab vedotin receives orphan drug status for Hodgkin } \\
\text { lymphoma in the US }\end{array}$ & 15 February 2007 \\
\hline 22 November 2006 & Phase Change & Phase I clinical trials in hematologic malignancies in US (IV) & 22 November 2006 \\
\hline 8 August 2006 & Regulatory Status & $\begin{array}{l}\text { Seattle Genetic Inc. has filed an IND with the US FDA for the } \\
\text { treatment of Hodgkin lymphoma and other CD-positive } \\
\text { hematologic malignancies }\end{array}$ & 8 August 2006 \\
\hline 13 September 2005 & Scientific Update & $\begin{array}{l}\text { Data presented at the 9th International Conference on } \\
\text { Malignant Lymphoma (ICML-2005) have been added to the } \\
\text { cancer pharmacodynamics section }{ }^{[34]}\end{array}$ & 13 September 2005 \\
\hline 15 June 2005 & Scientific Update & $\begin{array}{l}\text { Preclinical data from a media release have been added to the } \\
\text { cancer pharmacodynamics section }{ }^{[33]}\end{array}$ & 15 June 2005 \\
\hline 15 July 2004 & Licensing Status & $\begin{array}{l}\text { Seattle Genetics Inc. enters preferred provider agreement with } \\
\text { Albany Molecular Research for antibody-drug conjugate } \\
\text { manufacturing }{ }^{[2]}\end{array}$ & 15 July 2004 \\
\hline 12 September 2003 & Scientific Update & $\begin{array}{l}\text { A preclinical study has been added to the adverse events } \\
\text { section }^{[32]}\end{array}$ & 12 September 2003 \\
\hline 22 July 2003 & Scientific Update & $\begin{array}{l}\text { Data presented at the 94th Annual Meeting of the American } \\
\text { Association for Cancer Research (AACR-20-03) have been } \\
\text { added to the cancer pharmacodynamics and pharmacokinetics } \\
\text { section }^{[28]}\end{array}$ & 22 July 2003 \\
\hline 28 May 2003 & Phase Change & Preclinical trials in hematologic malignancies in the US (IV) & 28 May 2003 \\
\hline
\end{tabular}

patients with Hodgkin lymphoma and CD30positive hematologic malignancies. The majority of adverse events were grade 1 and 2, with the most common being fatigue, fever, diarrhea, nausea, and peripheral neuropathy. The maximum tolerated dose was defined as $1.8 \mathrm{mg} / \mathrm{kg}$. Patients received doses of brentuximab vedotin every 3 weeks, escalating from $0.1 \mathrm{mg} / \mathrm{kg}$ to $3.6 \mathrm{mg} / \mathrm{kg}$. Doselimiting toxicities of hyperglycemia, prostatitis, and neutropenic fever were observed at $2.7 \mathrm{mg} / \mathrm{kg}$. One patient treated at $3.6 \mathrm{mg} / \mathrm{kg}$ experienced fever, neutropenia, and sepsis, and died 14 days after the first dose of brentuximab vedotin. An additional two patients developed a positive anti-therapeutic antibody response to brentuximab vedotin in preliminary analyses. Less than $10 \%$ of brentuximab vedotin doses were delayed due to toxicity, primarily neutropenia, at higher doses. ${ }^{[18,20,29,30]}$

In an open-label, uncontrolled, phase I trial evaluating weekly dosing for refractory or relapsed lymphoma (Hodgkin or systemic ALCL), interim results for 35 evaluable patients showed that brentuximab vedotin was generally well tolerated. The majority of adverse events were grade 1 and 2, with the most common being fatigue, nausea, neutropenia, and peripheral neuropathy. Patients received weekly doses of $0.4-1.4 \mathrm{mg} / \mathrm{kg}$, for 3 of 4 weeks, for a minimum of two cycles. ${ }^{[22,23]}$

Preliminary data from 11 patients with Hodgkin lymphoma $(\mathrm{n}=9)$ and systemic ALCL (nonHodgkin lymphoma; $n=2$ ) who received retreatment with brentuximab vedotin monotherapy have shown that the drug was well tolerated in this setting. All drug-related adverse events were grade 1 or 2 , with the most common events being peripheral neuropathy, hair loss (alopecia), joint pain (musculoskeletal pain), and injection-site irritation. Patients were heavily pretreated with a range of 2-11 prior therapies. Five patients had received a prior ASCT. Patients had achieved 
Table III. Forecasts

\begin{tabular}{|c|c|c|c|}
\hline \multicolumn{4}{|c|}{ InThought Probability of Approval ${ }^{a}$} \\
\hline Indication & Approval Date Estimate & inThought Approvability Index & Last Update \\
\hline Hodgkin lymphoma & NE & $63 \%$ (NYR) & 10 Apr 2010 \\
\hline Non-Hodgkin lymphoma & $\mathrm{NE}$ & $31 \%$ (NYR) & 30 Jul 2009 \\
\hline \multicolumn{4}{|c|}{$\begin{array}{l}\text { The Wolters Kluwer Health Approvability Index is a dynamic tool that assesses the progress of a drug candidate through clinical } \\
\text { development, evaluating strength of clinical data and trial design, benchmarked against historical parameters and likelihood to maintain } \\
\text { forward momentum. Points are assigned for specific line items relating to safety, efficacy, and other factors in each phase of clinical } \\
\text { development. Possible points total } 100 \text { upon drug approval, and are allocated in each phase according to the historical approval rate of } \\
\text { similar drugs, such that the current points of a drug relate to its probability of approval. In addition, a letter grade is assigned and reflects the } \\
\text { momentum of a drug candidate in its current phase, with 'A' indicating significantly above average/likely to progress, 'C' indicating average, } \\
\text { and 'F' indicating significantly below average/unlikely to progress. 'NYR' stands for 'Not Yet Rated,' indicating that the probability of } \\
\text { approval is based on historical approval rates for similar drugs according to indication, molecule type, novelty, and phase, but without } \\
\text { analyses of clinical data, trial design, and other factors specific to the individual agent. }\end{array}$} \\
\hline
\end{tabular}

stable disease with decreasing tumor volume or better during prior treatment with brentuximab vedotin, discontinued treatment, and subsequently experienced disease progression. ${ }^{[31]}$

Animal Toxicology: In severe combined immunodeficiency (SCID) mouse xenograft models of ALCL or Hodgkin lymphoma, mice treated with brentuximab vedotin $30 \mathrm{mg} / \mathrm{kg}$ showed no signs of toxicity. ${ }^{[32]}$

\subsection{Pharmacodynamics}

\subsubsection{Cancer}

Preclinical: In vitro studies showed that the peptide linkage in brentuximab vedotin was efficiently cleaved by lyosomal proteases following CD30 binding and internalization, releasing the fully active drug compound, MMAE into the cell cytosols. This resulted in growth arrest in G2/M phase, apoptosis, and death. Brentuximab vedotin had potent cytotoxic activity against CD30expressing cells $(50 \%$ inhibitory concentration $\left[\mathrm{IC}_{50}\right]<10 \mathrm{ng} / \mathrm{mL}$ ) but was 300 -fold less potent against antigen-negative cells. In vivo studies showed that $80 \%$ of Hodgkin lymphoma and ALCL xenografted mice treated with brentuximab vedotin survived disease-free at doses as low as $1 \mathrm{mg} / \mathrm{kg}$. ${ }^{[28,32]}$

In preclinical models of ALCL, brentuximab vedotin displayed $\mathrm{IC}_{50}$ values ranging from 3.9 to $15.8 \mathrm{ng} / \mathrm{mL}$ against CD30+ cell lines, and an $\mathrm{IC}_{50}$ $>1000 \mathrm{ng} / \mathrm{mL}$ against the CD30 line WSU-NHL. In a SCID mouse model of ALCL, brentuximab vedotin exhibited dose-dependent antitumor ac- tivity, with complete regressions achieved using doses $\geq 0.5 \mathrm{mg} / \mathrm{kg}$ (with repeat dosing) and $\geq 1 \mathrm{mg} / \mathrm{kg}$ (with single dosing). ${ }^{[33,34]}$

Preclinical studies in animal models of Hodgkin lymphoma indicated that brentuximab vedotin localized in tumor tissue and had potent antitumor activity whether administered alone or in combination with chemotherapy. When combined with doxorubicin, bleomycin, vinblastine, and dacarbazine, or gemcitabine, the antitumor activity was markedly better than with brentuximab vedotin alone or chemotherapy alone. Combination therapy was not associated with alterations in CD30 expression in tumors. ${ }^{[35]}$

Preclinical data have demonstrated the superior antitumor activity of brentuximab vedotin in Hodgkin lymphoma, compared with that of several non-targeted drugs, including vinorelbine, vinblastine, and unconjugated MMAE. Due to the targeting ability of brentuximab vedotin, concentrations of MMAE within tumors were up to 30-fold higher than the non-targeted drugs. Also, MMAE concentrations in tumors were 1000 -fold greater than MMAE blood concentrations following brentuximab vedotin administration. ${ }^{[36]}$

\subsection{Therapeutic Trials}

\subsection{Cancer}

Phase III : In interim results from the ATHERA trial of brentuximab vedotin in patients at high risk of residual Hodgkin lymphoma following ASCT, $75 \%$ of patients achieved an objective response, including 34\% complete remissions, 
and $40 \%$ partial remissions. The median duration of response was 29 weeks by independent central review and 47 weeks by investigator assessment. Stable disease was observed in $22 \%$ of patients, of which $3 \%$ had progressive disease and one patient was not evaluable for response. Tumor reductions were achieved in $94 \%$ of patients. PFS among all patients was 25 weeks by independent review and 39 weeks by investigator assessment. PFS among patients achieving a complete remission and median overall survival had not yet been reached at a median follow-up of approximately 1 year. ${ }^{[9]}$

Phase II: Treatment with brentuximab vedotin caused tumor reductions in $94 \%$ of patients with relapsed or refractory Hodgkin lymphoma in a pivotal, open-label phase II trial $(n=102)$. Furthermore, $75 \%$ of patients had an objective response that lasted for $\geq 6$ months. Brentuximab vedotin $(1.8 \mathrm{mg} / \mathrm{kg})$ was administered every 3 weeks for up to a total of 16 doses. ${ }^{[10,14,37]}$

Results from a phase II single-arm clinical trial with brentuximab vedotin $(1.8 \mathrm{mg} / \mathrm{kg}$ every 3 weeks for up to 16 total doses) in 58 patients with relapsed or refractory systemic ALCL showed an objective response in $86 \%$ of patients. Complete remission was achieved in $53 \%$ of patients. The rate of partial remissions was $33 \%, 3 \%$ of patients had stable disease, and $5 \%$ had progressive disease. ${ }^{[4]}$

Phase I: In a phase I study, brentuximab vedotin lead to an objective response rate in evaluable patients of $46 \%$, with $29 \%$ achieving complete remission. Median duration of response to date was at least 16 weeks with 15 patients continuing treatment. The trial enrolled patients with relapsed or refractory CD30+ lymphomas $(n=37)$ and brentuximab vedotin was administered weekly for 3 weeks in 28-day treatment cycles at doses of $0.4-1.4 \mathrm{mg} / \mathrm{kg}$ (30 minute or 2 hour intravenous infusions). ${ }^{[26]}$

In a phase I trial of brentuximab vedotin among 44 evaluable patients with Hodgkin lymphoma and CD30+ hematologic malignancies, 17 patients achieved objective responses, including nine complete responses and eight partial responses. A total of 18 additional patients had stable disease and nine patients progressed. The median duration of response was 22 weeks, with
11 responses ongoing at the time results were published. Across all dose levels, $86 \%$ of the 42 patients who had at least one post-baseline assessment achieved reductions in tumor volume. Among 28 evaluable patients treated at doses of $1.2 \mathrm{mg} / \mathrm{kg}$ and higher, $54 \%$ achieved an objective response, including $32 \%$ with complete responses. Additionally, $93 \%$ of these patients achieved tumor reductions, and their mean PFS was greater than 6 months. Patients received doses of brentuximab vedotin every 3 weeks, escalating from $0.1 \mathrm{mg} / \mathrm{kg}$ to $3.6 \mathrm{mg} / \mathrm{kg} \cdot{ }^{[18,19,20,30]}$ A further update showed that the objective response rate (combining all dose levels) was $39 \%$ (based on investigator assessment) and $41 \%$ (based on independent review). In patients who received brentuximab vedotin at doses of $\geq 1.2 \mathrm{mg} / \mathrm{kg}$, the overall response rate was $54 \%$ (based on investigator assessment) and 57\% (based on independent review). The median duration of response was $\geq 7.3$ months and eight patients remained in ongoing response. ${ }^{[29]}$

In an open-label, uncontrolled, phase I trial evaluating weekly brentuximab vedotin dosing for refractory or relapsed CD30+ lymphoma (Hodgkin or systemic ALCL), interim results showed that 16 of 35 evaluable patients achieved objective responses (ten complete responses). Eleven patients had stable disease and three had progressive disease. The median duration of response is $\geq 16$ weeks, with 15 patients still receiving treatment. Patients received weekly doses of $0.4-1.4 \mathrm{mg} / \mathrm{kg}$, for 3 of 4 weeks, for a minimum of two cycles. ${ }^{[22,23]}$

Preliminary data from 11 patients with Hodgkin lymphoma $(\mathrm{n}=9)$ and systemic ALCL (nonHodgkin lymphoma; $n=2$ ) who were retreated with brentuximab vedotin monotherapy have shown that objective responses were achieved in seven retreated patients $(64 \%$ of patients, including two complete remissions and five partial remissions). Tumor reductions were observed in 10 of 11 retreated patients. All patients were heavily pretreated (i.e. $2-11$ prior therapies). Five patients had received a prior ASCT. Patients had achieved stable disease with decreasing tumor volume or better during prior treatment with brentuximab vedotin, discontinued treatment, 
and subsequently experienced disease progression. The time to objective response ranged from 5 to 15 weeks. Three patients showed stable disease and one had progressive disease. The duration of retreatment objective responses ranged from $<1$ week (retreatment ongoing), to $>58$ weeks. Based on the small sample size, no difference in duration of retreatment response between patients with Hodgkin lymphoma and non-Hodgkin lymphoma (ALCL) was observed. Retreatment is ongoing in three patients. ${ }^{[31]}$

\section{References}

1. Seattle Genetics, Inc., Millennium: The Takeda Oncology Company, Takeda Pharmaceutical Company Limited. Seattle Genetics, Inc., Millennium: The Takeda Oncology Company, announce strategic collaboration for novel late stage lymphoma program brentuximab vedotin (SGN-35). www.takeda.com, 15 Dec 2009 Media Release

2. Seattle Genetics, Inc. Seattle Genetics Establishes Preferred Antibody-Drug Conjugate Manufacturing Relationship with Albany Molecular Research, Inc. www.seattlegen etics.com, 13 Jul 2004 Media Release

3. Seattle Genetics, Inc. Seattle Genetics Launches Manufacturing Campaign with Albany Molecular Research. www.seattlegenetics.com, 05 May 2005 Media Release

4. Seattle Genetics, Inc, Millennium: The Takeda Oncology Company. Seattle Genetics and Millennium Report Positive Data from Phase II Trial of Brentuximab Vedotin (SGN-35) in Relapsed or Refractory ALCL at ASH Annual Meeting. www.takeda.co.jp, 07 Dec 2010 Media Release

5. Seattle Genetics, Inc. Seattle Genetics Receives Orphan Drug Designations for SGN-35 in the United States and Europe. www.seattlegenetics.com, 27 Jan 2009 Media Release

6. Seattle Genetics, Inc. Seattle Genetics Receives Orphan Drug Designations for its SGN-33 and SGN-35 Programs. www.seattlegenetics.com, 14 Feb 2007 Media Release

7. Seattle Genetics, Inc. Seattle Genetics Receives FDA Fast Track Designation for SGN-35 for the Treatment of Hodgkin Lymphoma. www.seattlegenetics.com, 31 Mar 2009 Media Release

8. Seattle Genetics, Inc., Takeda Pharmaceutical Company Limited, Millennium: The Takeda Oncology Company. Seattle Genetics, Takeda and Millennium Announce Initiation of Phase III AETHERA Trial of Brentuximab Vedotin (SGN-35) for Post-Transplant Hodgkin Lymphoma. www.seattlegenetics.com, 08 Apr 2010 Media Release

9. Seattle Genetics, Inc. Seattle Genetics and Millennium Report Positive Data from Pivotal Trial of Brentuximab Vedotin (SGN-35) in Relapsed or Refractory Hodgkin Lymphoma at ASH Annual Meeting. www.seattlegene tics.com, 06 Dec 2010 Media Release

10. Seattle Genetics, Inc, Millennium: The Takeda Oncology Company. Seattle Genetics and Millennium Announce Positive Top-Line Brentuximab Vedotin (SGN-35) Data from Pivotal Trial in Relapsed and Refractory Hodgkin Lymphoma. www.seattlegenetics.com, 27 Sep 2010 Media Release
11. Seattle Genetics, Inc. Seattle Genetics Initiates Brentuximab Vedotin (SGN-35) Retreatment Clinical Trial. www.seat tlegenetics.com, 25 Jul 2009 Media Release

12. Seattle Genetics, Inc. Seattle Genetics Reports Strong Pipeline Progress and Second Quarter 2009 Financial Results. www.seagen.com, 24 Jul 2009 Media Release

13. Seattle Genetics, Inc, Millennium Pharmaceuticals Inc. Seattle Genetics and Millennium Announce Initiation of Phase I Combination Clinical Trial of Brentuximab Vedotin (SGN-35) for Front-line Hodgkin Lymphoma. www.seattlegenetics.com, 04 Feb 2010 Media Release

14. Seattle Genetics, Inc, Millennium. Seattle Genetics and Millennium Announce Positive Top-Line Brentuximab Vedotin (SGN-35) Data from Phase II Trial in Relapsed or Refractory ALCL. www.seattlegenetics.com, 11 Oct 2010 Media Release

15. Seattle Genetics, Inc, Millennium: The Takeda Oncology Company.Seattle Genetics and Millennium Complete Enrollment of Brentuximab Vedotin (SGN-35) Phase II ALCL Trial. www.seattlegenetics.com, 24 May 2010 Media Release

16. Seattle Genetics, Inc. Seattle Genetics Initiates Phase II Trial of SGN-35 for Anaplastic Large Cell Lymphoma. www.seattlegenetics.com, 19 Jun 2009 Media Release

17. Seattle Genetics, Inc. Seattle Genetics Reports Development Progress and Third Quarter 2008 Financial Results. www. seattlegenetics.com, 24 Oct 2008 Media Release

18. Seattle Genetics, Inc. Seattle Genetics Reports Durable Objective Responses with SGN-35 in Lymphoma. www.seat tlegenetics.com, 07 Dec 2008 Media Release

19. Seattle Genetics, Inc. Seattle Genetics Presents Preclinical Data on Antibody-Drug Conjugate Programs. www.seat tlegenetics.com, 25 Oct 2008 Media Release

20. Seattle Genetics, Inc. Seattle Genetics Reports Positive Phase I Data with SGN-35 in Hodgkin Lymphoma. www. seattlegenetics.com, 06 Nov 2007 Media Release

21. An Intensive QT/QTc Study to Investigate the Effects of SGN-35 (Brentuximab Vedotin) on Cardiac Ventricular Repolarization in Patients With CD30-Positive Malignancies. Clinical Trial Profile

22. Fanale M, Bartlett NL, Forero-Torres A, et al. The Antibody-Drug Conjugate Brentuximab Vedotin (SGN-35) Induced Multiple Objective Responses in Patients with Relapsed or Refractory CD30-Positive Lymphomas in a Phase 1 Weekly Dosing Study. 51st Annual Meeting and Exposition of the American Society of Hematology: abstr. 2731, 5 Dec 2009. Available from URL: http://www.he matology.org [English]

23. Seattle Genetics, Inc. Seattle Genetics Reports Data from a Weekly Dosing Phase I Clinical Trial of SGN-35 in Lymphoma. www.seattlegenetics.com, 02 Jun 2009 Media Release

24. Seattle Genetics, Inc. Seattle Genetics Expands SGN-35 Clinical Program with Initiation of Second Phase I Trial. www.seattlegenetics.com, 29 Mar 2008 Media Release

25. Seattle Genetics, Inc. Seattle Genetics Expands AntibodyDrug Conjugate Patent Portfolio. www.seattlegenetics. com, 12 Mar 2010 Media Release

26. Seattle Genetics, Inc. Seattle Genetics Reports Positive Data from Phase I Weekly-Dosing Clinical Trial of Brentuximab 
Vedotin (SGN-35) in Lymphoma. www.seattlegenetics. com, 08 Dec 2009 Media Release

27. Younes A, Forero-Torres A, Bartlett NL, et al. Objective responses in a phase I dose-escalation study of SGN-35, a novel antibody-drug conjugate targeting CD30, in patients with relapsed or refractory Hodgkin lymphoma. 44th Annual Meeting of the American Society of Clinical Oncology: abstr. 8526, 30 May 2008. Available from URL: http://www.asco.org [English]

28. Francisco JA, Cerveny CG, Meyer DL, et al. SGN-35, an anti-CD30 antibody-drug conjugate with potent antitumor activity. 94th Annual Meeting of the American Association for Cancer Research: 149 (plus poster) abstr. R770, $11 \mathrm{Jul}$ 2003. USA [English]

29. Seattle Genetics, Inc. Seattle Genetics Reports Data from SGN-35 Every Three Week Dosing Phase I Trial in Lymphoma. www.seattlegenetics.com, 08 Jun 2009 Media Release

30. Seattle Genetics, Inc. Seattle Genetics Reports Multiple Complete and Partial Responses with SGN-35 in Patients with Lymphoma. www.seattlegenetics.com, 04 Jun 2008 Media Release

31. Seattle Genetics, Inc, Millennium: The Takeda Oncology Company. Seattle Genetics and Millennium Report Positive Data on Retreatment with Brentuximab Vedotin (SGN-35) in Lymphoma. www.seagen.com, 08 Jun 2010 Media Release
32. Francisco JA, Cerveny CG, Meyer DL, et al. cAC10vcMMAE, an anti-CD30-monomethyl auristatin E conjugate with potent and selective antitumor activity. Blood 2003; 102 (4): 1458-65. USA [English]

33. Seattle Genetics Inc. Seattle Genetics Presents Data from SGN-35 Program at International Conference on Malignant Lymphoma. www.seattlegenetics.com, 10 Jun 2005 Media Release

34. Barton J, Hamblett K, Cerveny C, et al. The anti-CD30 antibody-drug conjugate SGN-35 is a potent therapy for the treatment of CD30+ malignancies. Annals of Oncology 2005; 16 (Suppl. 5): 134. USA [English]

35. Gerber HP, Oflazoglu E, Kissler K, et al. Combination of the anti-CD30-auristatin-E antibody-drug conjugate SGN-35 with chemotherapy improves antitumor activity in Hodgkin lymphoma. 20th-EORTC-NCI-AACR Symposium on Molecular Targets and Cancer Therapeutics: 166 abstr. 523, 24 Oct 2008. Available from URL: http://www.ecco-org.eu USA [English]

36. Seattle Genetics, Inc. Seattle Genetics Presents Preclinical Data on Antibody-Drug Conjugate Programs at AACR. www.seattlegenetics.com, 23 Apr 2009 Media Release

37. American Society of Hematology. New Standards of Care and Novel Treatment Options for Several Forms of Lymphoma Unveiled. www.hematology.org, 06 Dec 2010 Media Release 